\title{
Sutilezas dos Vínculos Não-Holônomos
}

Subtleties of Nonholonomic Constraints

\author{
Nivaldo A. Lemos \\ Departamento de Física, Universidade Federal Fluminense \\ Av. Litorânea s/n, Boa Viagem, 24210-340 Niterói, RJ, Brazil
}

Recebido em 13 de novembro, 2002. Aceito em 25 de novembro, 2002.

\begin{abstract}
O tratamento de vínculos não-holônomos na dinâmica lagrangiana exige cautela, pois procedimentos válidos para vínculos holônomos nem sempre se aplicam a vínculos não-holônomos. Fazemos uma revisão didática das principais propriedades dos vínculos não-holônomos, com destaque para certas sutilezas raramente ou nunca discutidas nos textos de mecânica analítica. Chamamos a atenção, também, para as pouco conhecidas equações de Voronec, que evitam multiplicadores de Lagrange.
\end{abstract}

The treatment of nonholonomic constraints in Lagrangian dynamics requires caution, since procedures valid for holonomic constraints do not always apply to nonholonomic constraints. We make a didactic review of the main properties of nonholonomic constraints, with emphasis on certain subtleties seldom or never discussed in texbooks on analytical mechanics. We also call attention to the little known Voronec equations, which avoid Lagrange multipliers.

\section{Introdução}

Vínculos não-holônomos ocorrem com freqüência na mecânica, especialmente em problemas envolvendo rolamento sem deslizamento de corpos rígidos. Portanto, a dinâmica de sistemas submetidos a vínculos não-holônomos é um tópico importante de qualquer curso abrangente de mecânica analítica. Além de interessantes de um ponto de vista estritamente teórico, tais sistemas encontram inúmeras aplicações práticas [1].

Vínculos não-holônomos diferem em vários aspectos dos vínculos holônomos, e um de nossos propósitos principais é chamar a atenção para os cuidados que precisam ser tomados a fim de evitar erros decorrentes da inobservância de certas sutilezas características dos vínculos não-holônomos.

Há diversas maneiras de atacar o problema da construção das equações de movimento para sistemas nãoholônomos. Nossa discussão será limitada à formulação das equações de movimento no contexto do formalismo lagrangiano. Em particular, discutiremos duas maneiras distintas de obter as equações de movimento de sistemas nãoholônomos na dinâmica lagrangiana sem o emprego de multiplicadores de Lagrange, uma das quais culmina nas pouco conhecidas equações de Voronec. Dois exemplos, um meramente acadêmico e outro da dinâmica dos corpos rígidos, serão usados para ilustrar os resultados gerais.

\section{Vínculos não-holônomos}

Se $x_{1}, \ldots, x_{N}$ são coordenadas que descrevem as configurações (posições das partículas) de um determinado sistema mecânico, vínculos holônomos são relações funcionais da forma

$$
f_{l}\left(x_{1}, \ldots, x_{N}, t\right)=0, l=1, \ldots, p
$$

que definem as configurações acessíveis, isto é, as que são compatíveis com as restrições impostas ao sistema. Vínculos holônomos restringem as configurações possíveis, e qualquer configuração compatível com os vínculos corresponde a um conjunto de coordenadas generalizadas convenientemente escolhidas.

Sistemas mecânicos não-holônomos são aqueles sujeitos a pelo menos um vínculo não-holônomo. Vamos focalizar nossa atenção nos vínculos não-integráveis que dependem linearmente das velocidades, podendo, portanto, ser expressos na forma

$$
\sum_{k=1}^{n} a_{l k} \dot{q}_{k}+a_{l 0}=0, l=1, \ldots, p,
$$

onde $q_{1}, \ldots, q_{n}$ são coordenadas que descrevem as configurações do sistema e os coeficientes $a_{l k}$ e $a_{l 0}$ são funções somente de $q_{1}, \ldots, q_{n}, t$. Apesar da aparente falta de generalidade, a classe de vínculos (2) abarca a vasta maioria dos problemas de interesse físico. Como veremos a seguir, as equações (2) restringem tão somente os deslocamentos possíveis do sistema. 


\section{II.1 Integrabilidade}

A fim de ilustrar a maior parte das propriedades gerais dos vínculos não-holônomos, tomaremos como exemplo um sistema mecânico meramente acadêmico constituído por uma partícula de massa unitária movendo-se livremente num plano exceto pelo vínculo

$$
\dot{y}-x=0,
$$

onde $x, y$ são coordenadas cartesianas.

Para início de conversa, como é possível decidir se este vínculo é ou não holônomo? O vínculo (3) será holônomo se e somente se ele for integrável, e ele é integrável se e somente se existe um fator integrante não-nulo $h(x, y, t)$ tal que

$$
(\dot{y}-x) h=\frac{d G}{d t} \equiv \frac{\partial G}{\partial x} \dot{x}+\frac{\partial G}{\partial y} \dot{y}+\frac{\partial G}{\partial t}
$$

para alguma função $G(x, y, t)$. Caso $G$ e o fator integrante $h$ existam, a equação (4) e a equação de vínculo (3) permitem concluir que

$$
G(x, y, t)-C=0,
$$

onde $C$ é uma constante. Nessas circunstâncias, o vínculo (3) seria holônomo por ser equivalente a (5), que tem exatamente a forma (1).

Admitindo a existência da função $G$, somos forçados a concluir que

$$
\frac{\partial G}{\partial x}=0 \quad, \quad \frac{\partial G}{\partial y}=h \quad, \quad \frac{\partial G}{\partial t}=-h x .
$$

Da igualdade das derivadas parciais cruzadas da função supostamente existente $G$ deduzimos que

$$
\begin{gathered}
\frac{\partial^{2} G}{\partial x \partial y}=\frac{\partial^{2} G}{\partial y \partial x} \Longrightarrow \frac{\partial h}{\partial x}=0 \Longrightarrow h=h(y, t), \\
\frac{\partial^{2} G}{\partial x \partial t}=\frac{\partial^{2} G}{\partial t \partial x} \Longrightarrow h=0 .
\end{gathered}
$$

A inexistência de um fator integrante não-nulo estabelece que o vínculo (3) não é holônomo.

Há uma sutileza na caracterização de um sistema nãoholônomo: a existência de vínculos isoladamente nãointegráveis não garante que o sistema seja não-holônomo, pois os referidos vínculos podem revelar-se integráveis quando vistos em conjunto. Considere, por exemplo [1],

$$
\left(x^{2}+y^{2}\right) \dot{x}+x z \dot{z}=0,\left(x^{2}+y^{2}\right) \dot{y}+y z \dot{z}=0 .
$$

É fácil provar que cada um desses vínculos, tomado separadamente, não é integrável. No entanto, dividindo uma equação de vínculo pela outra e eliminado a variável tempo, deduz-se facilmente $d x / d y=x / y$ ou $y=C_{1} x$. Substituindo este último resultado e $\dot{y}=C_{1} \dot{x}$ na primeira das equações (8), resulta imediatamente $x \frac{d}{d t}\left(x^{2}+y^{2}+z^{2}\right)=$
0. Portanto, em conjunto, os vínculos separadamente nãointegráveis (8) são, na verdade, holônomos e equivalentes a

$$
y=C_{1} x, x^{2}+y^{2}+z^{2}=C_{2} .
$$

Em suma, para decidir se um sistema mecânico é ou não holônomo é necessário considerar todos os vínculos de uma só vez. Critérios gerais de holonomia para vínculos do tipo (2) podem ser encontrados na Seção I.7 de [1].

\section{II.2 Acessibilidade}

Embora as equações de vínculo contenham explicitamente as variáveis de configuração, vínculos não-integráveis não restringem de modo algum as configurações do sistema. Em outras palavras, todas as configurações são acessíveis [2]. Vamos ilustrar este resultado geral com o nosso exemplo acadêmico. Se for possível provar a existência de uma trajetória ligando dois pontos quaisquer do espaço de configuração e obedecendo ao vínculo, estará estabelecida a acessibilidade de todas as configurações. Sejam $(a, b)$ e $(c, d)$ dois pontos quaisquer do espaço de configuração do sistema. Seja $f(t)$ uma função diferenciável e defina $y=f(t)$ e $x=\dot{f}(t)$. Com esta escolha, o vínculo (3) é automaticamente satisfeito. Basta, agora, impor $\dot{f}(0)=$ $a, f(0)=b, \dot{f}\left(t_{0}\right)=c, f\left(t_{0}\right)=d$ para que fique estabelecida uma conexão entre as configurações $(a, b)$ em $t=0$ e $(c, d)$ em $t=t_{0}$ sem violação do vínculo. Ora, há uma infinidade de funções com os requisitos desejados. Por exemplo, a função polinomial

$$
\begin{aligned}
f(t) & =b+a t+\frac{3(d-b)-(c+2 a) t_{0}}{t_{0}^{2}} t^{2} \\
& +\frac{2(b-d)+(c+a) t_{0}}{t_{0}^{3}} t^{3}
\end{aligned}
$$

dá conta do recado.

Em síntese, vínculos não-holônomos caracterizam-se por restringir apenas os deslocamentos possíveis a partir de uma dada configuração, mantendo acessíveis todas as configurações.

\section{Vínculos não-holônomos no for- malismo lagrangiano}

Há diversos aspectos que merecem ser considerados no que se refere ao problema de construir equações de movimento para sistemas não-holônomos com base no formalismo lagrangiano.

\section{III.1 Método dos multiplicadores de Lagrange}

O método padrão de incorporar sistemas não-holônomos ao formalismo lagrangiano consiste em adotar como postulado o princípio de Hamilton e levar em conta os vínculos 
por meio de multiplicadores de Lagrange. Escrevendo os vínculos (2) na forma diferencial equivalente

$$
\sum_{k=1}^{n} a_{l k} d q_{k}+a_{l 0} d t=0, l=1, \ldots, p
$$

e levando em conta que os deslocamento virtuais $\delta q_{k}$ são executados a tempo fixo $(d t=0)$, a compatibilidade com os vínculos impõe as condições

$$
\sum_{k=1}^{n} a_{l k} \delta q_{k}=0, l=1, \ldots, p
$$

de modo que os $\delta q$ s não são todos independentes. Seja $L$ a lagrangiana do sistema escrita como se não houvesse vínculos de espécie alguma e seja $S=\int_{t_{1}}^{t_{2}} L d t$ a ação. Levando em conta as condições (12) no princípio de Hamilton por meio de multiplicadores de Lagrange $\lambda_{1}, \ldots, \lambda_{p}$, demonstra-se prontamente que as equações de movimento tomam a forma

$$
\frac{d}{d t}\left(\frac{\partial L}{\partial \dot{q}_{k}}\right)-\frac{\partial L}{\partial q_{k}}=\sum_{l=1}^{p} \lambda_{l} a_{l k} \quad, \quad k=1, \ldots, n
$$

Estas equações, juntamente com as equações de vínculo (2), permitem determinar as coordenadas generalizadas e os multiplicadores de Lagrange em função do tempo. Os multiplicadores de Lagrange, por sua vez, determinam as forças generalizadas de vínculo [3].

\section{III.2 Dispensando os multiplicadores de La- grange}

Os livros não costumam comentar que, se não estivermos interessados nas forças de vínculo mas apenas nas equações de movimento do sistema, podemos dispensar os multiplicadores de Lagrange completamente. Para isto, basta resolver as equações (12) para $p$ variações $\delta q_{k}$ em termos das demais e substituir na expressão de $\delta S=\delta \int_{t_{1}}^{t_{2}} L d t$. Como os $\delta q$ s que agora aparecem em $\delta S$ são mutuamente independentes, o argumento habitual utilizado para obter as equações de Lagrange a partir do princípio de Hamilton aplica-se sem modificação. Na maioria dos problemas de interesse físico a resolução das equações (12) é trivial, o que torna o método facilmente aplicável.

Vamos ilustrar essas idéias com o nosso modelo acadêmico, que tem por lagrangiana

$$
L=\frac{1}{2}\left(\dot{x}^{2}+\dot{y}^{2}\right),
$$

e para o qual a condição (12) reduz-se a

$$
\delta y=0 .
$$

Segue-se que

$$
\begin{aligned}
\delta S & =\delta \int_{t_{1}}^{t_{2}} \frac{1}{2}\left(\dot{x}^{2}+\dot{y}^{2}\right) d t \\
& =\int_{t_{1}}^{t_{2}}(\dot{x} \delta \dot{x}+\dot{y} \delta \dot{y}) d t=\int_{t_{1}}^{t_{2}}(-\ddot{x} \delta x-\ddot{y} \delta y) d t
\end{aligned}
$$

onde foi feita a costumeira integração por partes com o uso de $\delta \dot{x}=\frac{d}{d t} \delta x, \delta \dot{y}=\frac{d}{d t} \delta y$ e foram impostas as condições nos extremos $\delta x\left(t_{1}\right)=\delta x\left(t_{2}\right)=\delta y\left(t_{1}\right)=\delta y\left(t_{2}\right)=0$. Exigindo $\delta S=0$, usando (15) em (16) e levando em conta que que a variação $\delta x$ é arbitrária, resulta

$$
\ddot{x}=0 \text {. }
$$

O movimento é obtido integrando esta última equação juntamente com a equação de vínculo (3). O resultado é

$$
x=x_{0}+v_{0} t, y=y_{0}+x_{0} t+\frac{1}{2} v_{0} t^{2}
$$

onde $x_{0}, y_{0}$ e $v_{0}$ são constantes arbitrárias.

Como exemplo menos elementar e fisicamente mais relevante, considere uma moeda homogênea de massa $m$ e raio $R$ que rola sem deslizar e sem tombar numa rampa de inclinação $\alpha$. Sejam $x, y$ as coordenadas cartesianas do centro de massa da moeda, com o eixo $x$ horizontal e o eixo $y$ correndo ao longo da rampa e orientado para baixo. Se $\phi$ é o ângulo de rotação da moeda em torno de seu eixo de simetria e $\theta$ é o ângulo do plano da moeda com o eixo $y$, a lagrangiana é

$$
L=\frac{m}{2}\left(\dot{x}^{2}+\dot{y}^{2}\right)+\frac{m R^{2}}{4} \dot{\phi}^{2}+\frac{m R^{2}}{8} \dot{\theta}^{2}+m g y \operatorname{sen} \alpha .
$$

Os vínculos de rolamento são

$$
\dot{x}-R \dot{\phi} \operatorname{sen} \theta=0, \dot{y}-R \dot{\phi} \cos \theta=0 .
$$

Uma dedução detalhada desta lagrangiana e dos vínculos pode ser encontrada em [4], embora tal dedução seja irrelevante para nossos propósitos.

Nas presentes circunstâncias, as equações (12) tomam a forma

$$
\delta x-R \delta \phi \operatorname{sen} \theta=0, \quad \delta y-R \delta \phi \cos \theta=0 .
$$

A variação da ação escreve-se

$$
\delta S=\int_{t_{1}}^{t_{2}}\left(m \dot{x} \delta \dot{x}+m \dot{y} \delta \dot{y}+\frac{m R^{2}}{2} \dot{\phi} \delta \dot{\phi}+\frac{m R^{2}}{4} \dot{\theta} \delta \dot{\theta}+m g \delta y \operatorname{sen} \alpha\right) d t
$$


Integrando por partes e usando (21), o princípio de Hamilton torna-se

$$
\delta S=\int_{t_{1}}^{t_{2}}\left\{\left(-m \ddot{x} R \operatorname{sen} \theta-m \ddot{y} R \cos \theta-\frac{m R^{2}}{2} \ddot{\phi}+m g R \cos \theta \operatorname{sen} \alpha\right) \delta \phi-\frac{m R^{2}}{4} \ddot{\theta} \delta \theta\right\} d t=0 .
$$

Levando em consideração a arbitrariedade de $\delta \phi$ e $\delta \theta$, resultam as equações de movimento

$$
\begin{gathered}
\frac{m R^{2}}{2} \ddot{\phi}=-m R \ddot{x} \operatorname{sen} \theta-m R \ddot{y} \cos \theta+m g R \cos \theta \operatorname{sen} \alpha, \\
\frac{m R^{2}}{4} \ddot{\theta}=0 .
\end{gathered}
$$

Diferenciando as equações de vínculo (20) em relação ao tempo e substituindo em (24), obtém-se imediatamente

$$
\frac{3 m R^{2}}{2} \ddot{\phi}=m g R \cos \theta \operatorname{sen} \alpha
$$

As equações (25) e (26), em conjunto com as equações de vínculo (20), determinam univocamente o movimento da moeda.

\section{III.3 Substituição dos vínculos na lagrangiana}

O formalismo lagrangiano foi concebido para lidar com vínculos holônomos. A essência da abordagem lagrangiana consiste em introduzir coordenadas generalizadas $q_{1}, \ldots, q_{n}$ de tal modo que as equações de vínculo (1) sejam identicamente satisfeitas por $x_{1}(q, t), \ldots, x_{N}(q, t)$. Em seguida, substituindo $x_{1}(q, t), \ldots, x_{N}(q, t)$ na energia cinética $T$ e na energia potencial $V$, resulta uma lagrangiana $L=$ $T-V=L(q, \dot{q}, t)$ expressa exclusivamente em termos das coordenadas e velocidades generalizadas. Em suma, pela substituição dos vínculos na lagrangiana são eliminadas variáveis redundantes e a dinânica passa a ser descrita em termos de um conjunto menor de coordenadas mutuamente independentes. Uma pergunta natural, que não costuma ser respondida nos livros de mecânica analítica, é se esse processo de substituição de vínculos na lagrangiana para eliminar variáveis, que é absolutamente correto quando os vínculos são holônomos, também é válido para vínculos não-holônomos.

Consideremos novamente nosso exemplo acadêmico. A coordenada $y$ é completamente eliminada pela substituição do vínculo (3) na lagrangiana (14), dando lugar à lagrangiana reduzida

$$
\bar{L}=\frac{1}{2}\left(\dot{x}^{2}+x^{2}\right) .
$$

A equação de Lagrange fornecida por $\bar{L}$ é $\ddot{x}-x=0$. Sua solução geral é $x(t)=A e^{t}+B e^{-t}$, que difere completamente da solução correta (18).

No caso da moeda rolante, é possível formalmente eliminar $x$ da lagrangiana (19) utilizando (20), dando como resultado a lagrangiana reduzida

$$
\bar{L}=\frac{m R^{2}}{4}\left(1+2 \operatorname{sen}^{2} \theta\right) \dot{\phi}^{2}+\frac{m}{2} \dot{y}^{2}+\frac{m R^{2}}{8} \dot{\theta}^{2}+m g y \operatorname{sen} \alpha .
$$

A variação da ação reduzida $\bar{S}=\int \bar{L} d t$ é obtida sem dificuldade:

$$
\delta \bar{S}=\int\left\{-\delta \phi \frac{d}{d t} \frac{m R^{2}\left(1+2 \operatorname{sen}^{2} \theta\right) \dot{\phi}}{2}+(-m \ddot{y}+m g \operatorname{sen} \alpha) \delta y+m R^{2}\left(\dot{\phi}^{2} \operatorname{sen} \theta \cos \theta-\frac{\ddot{\theta}}{4}\right) \delta \theta\right\} d t .
$$

Com a variável $x$ eliminada, resta apenas o vínculo envolvendo $y$. Usando $\delta y=R \delta \phi \cos \theta$ e igualando a zero os coeficientes das variações independentes $\delta \theta$ e $\delta \phi$, resultam as equações

$$
\ddot{\theta}-4 \dot{\phi}^{2} \operatorname{sen} \theta \cos \theta=0
$$

$\frac{d}{d t}\left[\frac{m R^{2}}{2}\left(1+2 \operatorname{sen}^{2} \theta\right) \dot{\phi}\right]=-(m \ddot{y}-m g \operatorname{sen} \alpha) R \cos \theta$.

Estas equações são completamente diferentes das equações de movimento corretas. Note, em particular, a discrepância entre (30) e (25), que é a equação correta para $\theta$.

Estes exemplos não deixam margem para dúvida: em geral não é permitido introduzir vínculos não-holônomos na lagrangiana para reduzir o número de variáveis mantendo as equações de movimento na forma lagrangiana padrão. Uma discussão minuciosa do princípio variacional de Hamilton permite identificar a origem da dificuldade. 


\section{III.4 Trajetórias de comparação no princípio variacional de Hamilton}

Quando os vínculos são holônomos, as trajetórias variadas obtidas por deslocamentos virtuais de trajetórias cinematicamente admissíveis também são cinematicamente admissíveis. Em outras palavras, se uma trajetória obedece às equações de vínculo, deslocamentos virtuais conduzem a trajetórias que também obedecem às equações de vínculo. Quando os vínculos não são holônomos, sabe-se que isto não é verdade em geral [5].

Esse fenômeno é de fácil verificação em nosso exemplo acadêmico. Um deslocamento virtual caracteriza-se, segundo a equação (15), por $\delta y=0$. Seja, agora, $(x, y)$ uma trajetória que obedece à equação (3) e seja $(\bar{x}, \bar{y})$ uma tra- jetória vizinha obtida mediante um deslocamento virtual, a saber

$$
\bar{x}=x+\delta x \quad, \quad \bar{y}=y+\delta y .
$$

Como conseqüência de (15),

$$
\dot{\bar{y}}-\bar{x}=\dot{y}-(x+\delta x)=-\delta x \neq 0,
$$

de modo que todas as trajetórias variadas violam o vínculo.

No caso da moeda rolante, o deslocamento virtual mais geral possível é

$$
\begin{aligned}
& \bar{x}=x+R \operatorname{sen} \theta \delta \phi, \bar{y}=y+R \cos \theta \delta \phi, \\
& \bar{\theta}=\theta+\delta \theta, \bar{\phi}=\phi+\delta \phi,
\end{aligned}
$$

onde usamos (21). Para a trajetória variada temos

$$
\dot{\bar{x}}-R \dot{\bar{\phi}} \operatorname{sen} \bar{\theta}=\dot{x}+R \delta \dot{\phi} \operatorname{sen} \theta+R \dot{\theta} \delta \phi \cos \theta-R(\dot{\phi}+\delta \dot{\phi})(\operatorname{sen} \theta+\cos \theta \delta \theta)
$$

Usando (20) e desprezando infinitésimos de segunda ordem, resulta

$$
\dot{\bar{x}}-R \dot{\bar{\phi}} \operatorname{sen} \bar{\theta}=R \dot{\theta} \cos \theta \delta \phi-R \dot{\phi} \cos \theta \delta \theta
$$

Devido à arbitrariedade de $\delta \theta$ e $\delta \phi, \dot{\bar{x}}-R \dot{\bar{\phi}}$ não é zero em geral e o vínculo é violado.

Tendo em vista que vínculos não-holônomos restringem apenas os deslocamentos possíveis, o presente estado de coisas é muito bem caracterizado por Whittaker [6] nos seguintes termos: "há infinitamente mais posições adjacentes possíveis do que deslocamentos possíveis a partir de uma dada posição."

O fato de as trajetórias variadas violarem os vínculos ajuda a entender porque não é permitido introduzir vínculos não-holônomos na lagrangiana para eliminar variáveis. Consideremos novamente nosso exemplo acadêmico. Quando se substitui $\dot{y}$ por $x$ na lagrangiana (14) se produz um problema variacional inequivalente no qual $\delta \dot{y}=\delta x \neq 0$, ao passo que no princípio variacional fisicamente correto tem-se necessariamente, em virtude da definição de variação, $\delta \dot{y}=\frac{d}{d t} \delta y=0$.

De maneira geral, a eliminação de variáveis da lagrangiana por meio de vínculos não-holônomos reduz indevidamente o número de graus de liberdade configuracionais, conduzindo a um problema variacional num número menor de variáveis e restrito apenas às trajetórias de comparação que satisfazem as equações de vínculo usadas no processo de eliminação - portanto, essas trajetórias variadas não podem podem ser obtidas da trajetória real por deslocamentos virtuais. Esse problema variacional modificado não é equivalente à versão original do princípio de Hamilton sujeito ao conjunto completo de vínculos não-holônomos. Isto explica por que não se deve inserir vínculos não-holônomos na lagrangiana com o propósito de reduzir o número de graus de liberdade configuracionais.

\section{III.5 Equações de Voronec}

A discussão anterior estabelece apenas que é proibido eliminar variáveis da lagrangiana por meio de vínculos não-holônomos se se quiser preservar a forma lagrangiana padrão das equações de movimento. Para certas formas freqüentemente assumidas pelas equações de vínculo, o referido processo de eliminação pode ser executado, porém as equações de movimento corretas não são as equações de Lagrange usuais, mas as equações de Voronec [1]. Uma vez que as equações de Voronec parecem ser pouco conhecidas, vamos apresentá-las em sua forma mais geral (o leitor é remetido a [1] para uma dedução).

Consideremos um sistema mecânico descrito pelas variáveis de configuração $q_{1}, \ldots, q_{n}$ e por um conjunto de vínculos não-holônomos que dependem linear e homogeneamente das velocidades. Suponhamos que as primeiras $m$ velocidades sejam mutuamente independentes, permitindo exprimir as $k=n-m$ velocidades restantes em termos das velocidades independentes. Isto significa que as equações de vínculo podem ser escritas na forma

$$
\dot{q}_{m+l}-\sum_{j=1}^{m} a_{l j} \dot{q}_{j}=0, l=1, \ldots, k
$$

onde os coeficientes $a_{l j}$ são funções das coordenadas generalizadas $q_{1}, \ldots, q_{n}$.

Seja $L(q, \dot{q}, t)$ a lagrangiana escrita ignorando completamente os vínculos não-holônomos (37). Eliminando as $k$ 
últimas velocidades da lagrangiana por meio das equações (37), resulta uma lagrangiana reduzida $\bar{L}$ :

$L\left(q_{1}, \ldots, q_{n}, \dot{q}_{1}, \ldots, \dot{q}_{n}, t\right)=\bar{L}\left(q_{1}, \ldots, q_{n}, \dot{q}_{1}, \ldots, \dot{q}_{m}, t\right)$

As equações de movimento de Voronec são [1]

$$
\frac{d}{d t}\left(\frac{\partial \bar{L}}{\partial \dot{q}_{i}}\right)-\frac{\partial \bar{L}}{\partial q_{i}}=\sum_{\nu=1}^{k} \frac{\partial \bar{L}}{\partial q_{m+\nu}} a_{\nu i}+\sum_{\nu=1}^{k} \sum_{j=1}^{m} \frac{\partial L}{\partial \dot{q}_{m+\nu}} b_{i j}^{\nu} \dot{q}_{j}, \quad i=1, \ldots, m
$$

onde

$$
b_{i j}^{\nu}=\frac{\partial a_{\nu i}}{\partial q_{j}}-\frac{\partial a_{\nu j}}{\partial q_{i}}+\sum_{\mu=1}^{k}\left(\frac{\partial a_{\nu i}}{\partial q_{m+\mu}} a_{\mu j}-\frac{\partial a_{\nu j}}{\partial q_{m+\mu}} a_{\mu i}\right)
$$

Note que as equações (39) também envolvem a lagrangiana original $L\left(q_{1}, \ldots, q_{n}, \dot{q}_{1}, \ldots, \dot{q}_{n}, t\right)$. Quando os vínculos (37) são integráveis (logo holônomos) $a_{l j}=$ $\partial f_{l}\left(q_{1}, \ldots, q_{m}\right) / \partial q_{j}$, de modo que o lado direito de (39) é zero porque os coeficientes $b_{i j}^{\nu}$ são identicamente nulos e $\bar{L}$ depende unicamente das primeiras coordenadas $q_{1}, \ldots, q_{m}$. Neste caso, as equações de Voronec coincidem com as equações de Lagrange em termos da lagrangiana reduzida $\bar{L}$ apenas, como esperado.

À guisa de aplicação, retomemos o problema da moeda rolante. Fazendo $q_{1}=\phi, q_{2}=\theta, q_{3}=x, q_{4}=y$, os vínculos (20) podem ser postos na forma (37) com

$$
a_{11}=R \operatorname{sen} \theta, a_{12}=0, a_{21}=R \cos \theta, a_{22}=0 .
$$

No presente caso, $m=k=2$.

A lagrangiana reduzida, obtida por inserção das equações de vínculo (20) na lagrangiana original (19), é

$$
\bar{L}=\frac{3 m R^{2}}{4} \dot{\phi}^{2}+\frac{m R^{2}}{8} \dot{\theta}^{2}+m g y \operatorname{sen} \alpha .
$$

Uma aplicação direta da definição (40) fornece os únicos coeficientes não-nulos $b_{i j}^{\nu}$ :

$$
b_{12}^{1}=-b_{21}^{1}=R \cos \theta, b_{12}^{2}=-b_{21}^{2}=-R \operatorname{sen} \theta .
$$

Assim, as equações de Voronec para a moeda rolante são

$$
\frac{3 m R^{2}}{2} \ddot{\phi}=m g R \operatorname{sen} \alpha \cos \theta+m \dot{x} R \dot{\theta} \cos \theta-m \dot{y} R \dot{\theta} \operatorname{sen} \theta \text {, }
$$

$$
\frac{m R^{2}}{4} \ddot{\theta}=-m \dot{x} R \dot{\phi} \cos \theta+m \dot{y} R \dot{\phi} \operatorname{sen} \theta
$$

Lançando mão das equações de vínculo (20), estas equações de movimento tomam a forma substancialmente mais simples

$$
\ddot{\phi}=\frac{2 g \operatorname{sen} \alpha}{3 R} \cos \theta \quad, \quad \ddot{\theta}=0 .
$$

Estas últimas equações coincidem precisamente com (25) e (26). Comprova-se, assim, que as equações de Voronec descrevem corretamente o movimento da moeda rolante.

Como regra geral, se as equações de vínculo são funções lineares e homogêneas das velocidades, a substituição dos vínculos não-holônomos na lagrangiana é permitida, mas as equações de Lagrange têm que ser substituídas pelas equações de Voronec.

\section{Conclusão}

Da discussão anterior algumas lições podem ser extraídas. Embora as equações que exprimem os vínculos não-holônomos contenham explicitamente as variáveis de configuração, apenas os deslocamentos possíveis são restringidos, todas as configurações permanecendo acessíveis. A caracterização de um sistema como não-holônomo exige a consideração da totalidade dos vínculos, pois vínculos separadamente não-holônomos podem ser holônomos quando tomados em conjunto. Quando não há interesse na determinação das forças de vínculo, os multiplicadores de Lagrange podem ser dispensados, bastando para tal substituir as variações dependentes em termos das variações independentes no princípio de Hamilton e utilizar os argumentos habituais aplicáveis a sistemas sem vínculos. $\mathrm{Na}$ presença de vínculos não-holônomos, as trajetórias variadas no princípio de Hamilton não são cinematicamente admissíveis, isto é, violam os vínculos. Por esse motivo, vínculos não-holônomos não podem ser substituídos na lagrangiana para eliminar variáveis mantendo intactas as equações de Lagrange. Quando os vínculos são funções lineares e homogêneas das velocidades generalizadas, tal 
substituição é possível, mas as equações de movimento corretas são as equações de Voronec.

\section{Referências}

[1] Ju. I. Neı̆mark e N. A. Fufaev, Dynamics of Nonholonomic Systems (American Mathematical Society, Providence, Rhode Island, 1972).

[2] L. A. Pars, A Treatise on Analytical Dynamics (Ox Bow Press, Woodbridge, CT, 1965), Cap. 1.
[3] H. Goldstein, Classical Mechanics (Addison-Wesley, Reading, MA, 1980), $2 \underline{a}$ edição, Cap. 2.

[4] N. A. Lemos, Mecânica Analítica. Notas de Aula, Departamento de Física da Universidade Federal Fuminense (2002).

[5] H. Rund, The Hamilton-Jacobi Theory in the Calculus of Variations (Robert E. Krieger, Huntington, NY, 1973), Cap. 5, Seção 5.

[6] E. T. Whittaker, A Treatise on the Analytical Dynamics of Particles and Rigid Bodies (Dover, New York, NY, 1944), Cap. IX, Seção 102. 